\title{
The Power of Power Law: A View from VoD Service
}

\author{
Hoon Lee
}

\begin{abstract}
In this work we investigate the power of power law in the $\mathrm{VoD}$ service over IP network. Especially, we show that the power of power law is strong in the location of the contents storage for the VoD service and its impact on the link dimensioning. This work first shows the instance of the power law in the popularity of the video contents from the real data for the IPTV service. After that we propose an analytic model that characterizes the behavior of customers in accessing the video servers as well as the power law in the viewing pattern. Finally, we show the impact of the power law by proposing a model for the cost as well as link dimensioning. Via numerical experiment we show the implication of the proposition.
\end{abstract}

Key Words : Internet, VoD, Power law, Link dimensioning, Cost performance

\section{I . Introduction}

Recently, the IPTV (Internet Protocol TV) service is very popular and it is the most important killer application and source of cash to the Internet service providers (ISPs). VoD (Video on Demand) became a companion service to the IPTV service. It is envisioned that $\mathrm{VoD}$ is popular due to merits such as time-disjoint property of viewing, abundant choice of contents, and diverse applications such as stock market, music video, sports games, dramas, etc.

One of the most important problems in providing the VoD service to mass customers is the determination for the location of the video program. Video programs are located at servers in various ways: centralized or distributed, the operation of which is described in detail later. This work promotes the distributed architecture for the location of video servers, because it is much efficient.

On the other hand, it is usually known that one can find the phenomenon of power law from measurements in the Internet such as a growth model, caching, and networking ${ }^{[1]}$. The definition of power law is as follows: the probability mass function (pmf) of a random event to occur is represented by a power function, a detailed definition of which is given at Section 2 .

Breslau et al. argue that the frequency of the web-object follows power law ${ }^{[2]}$. Mitzenmacher shows the instance of power law in the size of the web-object $^{[3]}$. It is known that the number of online friends in the SNS (Social Network Service) follows the power law ${ }^{[4]}$.

When it comes to the video streaming service, lots of works argue the existence of the power law in the viewing pattern of the video. Gill et al. show that the popularity of the YouTube video follows a Zipf-like distribution, where power law is synonymous with Zipf's law ${ }^{[5]}$. Cha et al. show that the popularity of the TV channel in the IP network follows the Zipf-like property, too ${ }^{[6]}$.

When it comes to the link dimensioning, there exists a few literature that deals with the power law. Won et al. assume that the popularity of the IPTV program follows a Zipf's law with a binary exponential function where the number of customers for (i-1)-th popular program is twice greater than

※ This work is supported by Changwon National University 2012-2014 research project.

- First Author : Dept. of Information \& Communication Engineering, Changwon National University, hoony@cwnu.ac.kr, Lifelong Member Paper number : KICS2013-04-175, 접수일자 : 2013년 4월 16일, 최종논문접수일자 : 2013년 7월 24일 
that of i-th popular program ${ }^{[7]}$. However, in reality, the popularity of the IPTV program does not follow the binary exponential function.

Lee proposed an analytic model to exploit the power law in the determination of the required capacity of links for the VoD servers with three-level hierarchy ${ }^{[8]}$. In [8] Lee assumed two opposite architectures (centralized and distributed) for the location of the video servers, and showed that one can save bandwidth from the distributed architecture. Lee assumed an arbitrary function for the power law (at that time he had no real-field data about the popularity model for the VoD service, yet) and he used an $\mathrm{M} / \mathrm{M} / \mathrm{c} / \mathrm{c}$ queue to evaluate the performance of the media server.

However, $\mathrm{M} / \mathrm{M} / \mathrm{c} / \mathrm{c}$ queue has a drawback in modeling the VoD server because it estimates the performance of the system too pessimistically. The $\mathrm{M} / \mathrm{M} / \mathrm{c} / \mathrm{c}$ queue model assumes that the system has a limited server capacity for the $\operatorname{VoD}$ storage system. However, it is not realistic at the present, because the server capacity as well as the link capacity is almost unlimited. Therefore, a more realistic queuing model that reflects the real situation of the current VoD system has to be introduced, which is given in Section IV.

This work aims at the proposition of an analytic model to investigate the relationship between the location of the distributed video servers and the performance of the system concerning the link dimensioning and cost. To that purpose, let us exploit the power law in locating the video servers in a distributed fashion, from which we investigate its effect in link dimensioning and cost. To the best of author's knowledge, this work is the first attempt to investigate the relationship between the power law in video popularity and its performance evaluation in the link dimensioning and cost.

This work is composed as follows: In Section II, we describe the generic attributes of VoD service. In Section III, we present the instance of power law in the probability of viewing the video program. In Section IV, we apply the power law in locating the video program to a distributed storage servers, from which we propose a method to estimate the capacity of the link and its cost. In Section V, we show the result of the numerical experiment. In Section VI, we summarize this work.

\section{VoD service and its attributes}

VoD is a service for the viewing of a video by on-demand, so it is provided by a unicast transfer of packets from the video server (VS) to the customer's UE (User Equipment). When a customer requests a video program that is stored in $\mathrm{VS}$, the video content is transferred to the customer's UE. Network architecture for the $\mathrm{VoD}$ service varies depending on ISPs. Generally speaking, there are three distinct areas in the network that support the VoD service: contents provider (CP) where video contents are located, ISP that connects end user (EU) and CP, and end users ${ }^{[9]}$.

Let us briefly summarize the network architecture for the VoD service. End users as well as CP are connected to a broadband IP network via an access network such as DSL (Digital Subscriber's Line), optical or wireless access network. Distribution network aggregates a number of neighboring access networks, and backbone (or core) network supports transfer of packets between distribution networks that are located throughout geographically distributed locations.

There exist a number of methods to locate video server: ranging from centralized server model to distributed server model. The simplest way is a centralized server model in which there is a single storage system at the CP. Centralized server model has advantages and drawbacks. The simplicity in the management of the contents is most advantageous. There needs no remote update of the storage. However, there are a number of drawbacks when a nationwide service is assumed for the $\mathrm{VoD}$ service, because even the largest centralized server cannot scale to large number (e.g., millions) of subscribers. In addition, excessive traffic between $\mathrm{CP}$ and customers is most disadvantageous.

Recently, a total separation of storage from streaming is a trend for large-scale VoD services, where the most popular content is located at the 
edge of the network and less popular content is transferred from a neighboring server, and the original programs are located at the central storage, via which scaling problem and traffic overload can be resolved. This results in a distributed server location.

As to the distributed server model, the mostly adopted scheme is a hierarchical location of video contents into a number of servers in a geographically distributed location such that central server has a complete list of video contents, whereas the other servers have a few popular video contents.

The number and location of the servers are determined by a policy of the ISP. In [8, 10] a tri-level model for the location of servers is proposed. However, one can extend the level without loss of generality. In this work we assume that there are three types of servers in the network: central server (CS), intermediate server (IS), and local server (LS).

CS is located at the CP-side of the network (at the core network), LS is located at the EU-side (at the access network), and IS is located in the middle (at the distribution network). Spagna et al. have illustrated a schematic diagram for a structure of a mobile carrier network with distributed content cache servers in [18]. This work refers to Spagna's architecture as a basic framework for VoD service. If we depict those elements in an abstract manner we obtain a network model that is given in Fig.1.

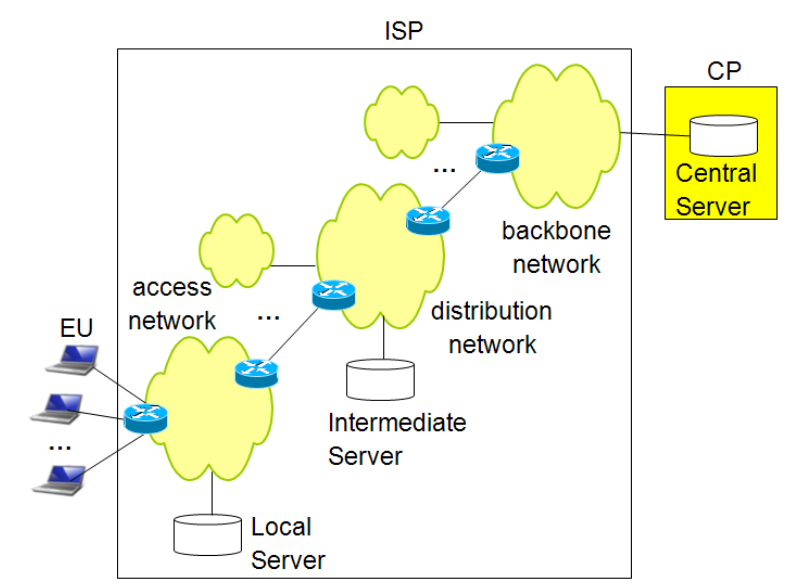

Fig. 1. An architecture for $\mathrm{VoD}$ services

For the storage of video contents we propose the following policy: LS has the most popular $\mathrm{k}_{1}$ video programs. IS has the next popular $\mathrm{k}_{2}$ video programs as well as the most popular $\mathrm{k}_{1}$ video programs. Finally, CS has $100 \%$ of video contents, the number of which is assumed to be $\mathrm{M}$.

In this case, customers' requests for video contents are first processed at the LS where the most popular $\mathrm{k}_{1}$ video programs are located. If there is no match for video contents at the LS, the request is transferred to the IS at which the additional video contents in the popularity level from $\mathrm{k}_{1}+1$ to $\mathrm{k}_{1}+\mathrm{k}_{2}$ are stored. If there is no match for video contents at the IS, the request is transferred to the CS where M video contents are stored. It is usually known that there does not exist an optimal solution for the determination of $\mathrm{k}_{1}$ and $\mathrm{k}_{2}$ in an analytic method, instead only a numerical experiment exists ${ }^{[10]}$.

\section{Power law in viewing popularity}

First, let us define power law. To do that, we have to define a power function. Power function (let us denote it $\mathrm{f}(\mathrm{x}))$ is a scale-invariant function of the form $\mathrm{f}(\mathrm{x})=\alpha \mathrm{x}^{-\beta}$, where $a$ and $\beta$ are positive constants that represents normalization and scaling exponent, respectively ${ }^{[11]}$.

If we take logarithm on both sides of the function $f(x)$, we have the following formula:

$$
\log f(x)=-\beta \log x+\log \alpha
$$

As we can see from (1), the function appears as a straight line when it is plotted on a log-log scale, which is a distinctive feature of the power law.

When a distribution of a data set is represented as a power function, especially when the random variable is large, it is said to follow a power law. Power law is usually called a Zipf's law, which is named after a linguist George K. Zipf who found this law $^{[12]}$.

It is usually known that there exists power law in the distribution of the popularity for the viewing pattern of the VoD service ${ }^{[8]}$. A few video programs are very popular, whereas a large number of video programs are viewed by a few customers, but it is not negligible. This is called long-tail ${ }^{[12]}$. 
Let us show real data that illustrates the power law. Fig.2 illustrates the number of download for the top 100 programs of an IPTV service (including VoD) at an ISP in Korea ${ }^{[13]}$.

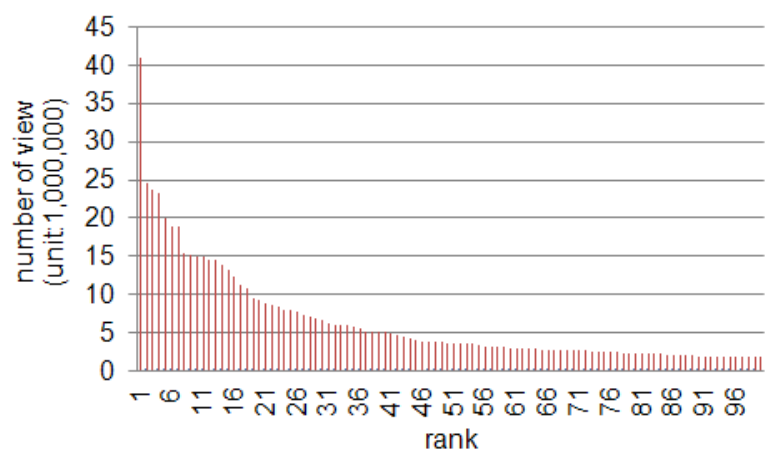

Fig. 2. Popularity of the contents (flat scale)

As one can find from the figure, there exists a long tail for the distribution of the popularity. From the figure one can compute a pmf $p_{i}$ (the lower index $\mathrm{i}$ is the rank in the popularity, $1 \leqq \mathrm{i} \leqq 100, \mathrm{p}_{1}$ being the most popular and $p_{100}$ being the least popular) for the popularity of the download of the video program by using a statistical computation or curve fitting algorithm, eg. ${ }^{[14]}$. Note that the pmf obtained by a curve fitting has a closed-form formula, which may be convenient in representing the power law in a closed formula. However, statistical computation is more accurate than that by a curve fitting. Therefore, we use a statistical computation method for pmf. For example, $\mathrm{p}_{1}=0.0639, \mathrm{p}_{2}=0.0384, \mathrm{p}_{50}=0.0058$, and $\mathrm{p}_{100}=0.0027$ in Fig.2. Note that $p_{i}$ does not follow a binary exponent.

\section{Application to link dimensioning}

In order to investigate the impact of the power law in the design of the network such as the link dimensioning, let us carry out an analysis for the system.

\subsection{System model}

Let us compute a probability for the number of customers in the system at equilibrium. Let $\mathrm{K}$ be the number of customers at an access network who enjoy a $\mathrm{VoD}$ service. When $\mathrm{K}$ is sufficiently large and the behavior of customers is independent, we can assume that the arrival of customers follows a Poisson distribution ${ }^{[12]}$. Let $\lambda$ be the mean arrival rate of the request for the video program.

Now let us describe a model for the service time (alias, holding time) of a flow. It is known in [15] that the mean holding time of $\mathrm{VoD}$ is very short: about $50 \%$ of the sessions have a holding time less than 8 minutes and only $13 \%$ of the sessions survive more than 50 minutes. From this observation let us assume that the mean holding time of a session follows exponential distribution with mean $1 / \mu$, which results in a mean system load of $\rho=\lambda / \mu$.

Let us assume that the system has enough service capacity (computing power and link capacity), and the server has a sufficient buffer space to accommodate the customers, which is usual at the current system. Then we can model the system as an $\mathrm{M} / \mathrm{M} / \infty / / \mathrm{K}$ queue, where ' $/ / \mathrm{K}$ ' represents that the number of customer is finite with $\mathrm{K}$.

For the $\mathrm{M} / \mathrm{M} / \infty / / \mathrm{K}$ queue we have the following formulae for the arrival and departure of customers at system state $\mathrm{i}^{[16]}$ :

$$
\begin{aligned}
& \lambda_{i}= \begin{cases}\lambda(K-i) & 0 \leq i \leq K \\
0 & \text { else }\end{cases} \\
& \mu_{i}=i \mu, 1 \leq i \leq K
\end{aligned}
$$

Let $\pi(i)$ be the probability that the system is in state $\mathrm{i}(0 \leqq \mathrm{i} \leqq \mathrm{K})$ in equilibrium. By using the analysis of birth and death process in queuing theory [16], we obtain the following formula for $\pi(i)$.

$$
\pi(i)=\left(\begin{array}{c}
K \\
i
\end{array}\right) \frac{\rho^{i}}{(1+\rho)^{K}}
$$

\subsection{Link dimensioning}

Referring to Fig.1, one can find that the required capacity of the link in the end-to-end path of a video program from the video server to the UE depends on the power law property of the viewing pattern from the customers. Therefore, let us focus 
on the capacity of the link between neighboring routers that take care of each video server.

For the simplicity let us assume that the capacity of the link for the VoD service is defined as a unit of channel, where a channel is the unit of a link that carries a stream of packets for a single flow. This is called as a flow-aware model for the link dimensioning. In [17], Lee proposed a flow-aware link dimensioning method for the guaranteed-QoS service by matching the packet-level aggregated big-pipe model into an equivalent per-flow link model. This analogy fits very well to the VoD service with homogeneous types of contents, because the data rate of a packet stream for the $\mathrm{VoD}$ service is almost constant or statistically stationary with constant effective bandwidth (EB), so that the unit of the bandwidth required by a flow can be assumed to be almost fixed (See [17] for a detailed discussion about EB).

First, let us compute the probability $\mathrm{P}_{\mathrm{LS}}, \mathrm{P}_{\mathrm{IS}}$, and $\mathrm{P}_{\mathrm{CS}}$ that a users' request from an access network for video contents is served in LS, IS, and CS, respectively, which is given as follows:

$$
\begin{aligned}
P_{L S} & =\sum_{i=1}^{k_{1}} p_{i} \\
P_{I S} & =\sum_{i=k_{1}+1}^{k_{1}+k_{2}} p_{i} \\
P_{C S} & =\sum_{i=k_{1}+k_{2}+1}^{M} p_{i}
\end{aligned}
$$

Let $\mathrm{L}_{\mathrm{CI}}, \mathrm{L}_{\mathrm{IL}}$, and $\mathrm{L}_{\mathrm{LU}}$ be the required capacity of link between the routers that manage the traffic between CS and IS, IS and LS, and LS and UE, respectively. Here, the capacity of the link indicates the number of channel, the capacity of which is determined by the required bandwidth for each video stream. Then, $\mathrm{L}_{\mathrm{CI}}, \mathrm{L}_{\mathrm{IL}}$, and $\mathrm{L}_{\mathrm{LU}}$ are represented as follows, where $\lceil\mathrm{x}\rceil$ is a ceiling function defined by a minimum integer not smaller than $\mathrm{x}$ :

$$
\begin{aligned}
& L_{C I}=\left\lceil\sum_{j=1}^{K} P_{C S} j \pi(j)\right\rceil \\
& L_{I L}=\left\lceil\sum_{j=1}^{K}\left(P_{I S}+P_{C S}\right) j \pi(j)\right\rceil \\
& L_{L U}=\left\lceil\sum_{j=1}^{K}\left(P_{L S}+P_{I S}+P_{C S}\right) j \pi(j)\right]
\end{aligned}
$$

Note that the capacity of link for the higher hierarchy is much smaller than that of the lower one. That is to say, $\mathrm{L}_{\mathrm{Cl}}<\mathrm{L}_{\mathrm{IL}}<\mathrm{L}_{\mathrm{LU}}$. This phenomenon is very desirable seen from the ISP, because the cost of delivering a video program over the backbone network is much greater than the delivery of a video program over the community network ${ }^{[10]}$.

\subsection{Analysis of cost}

As we have argued before, the cost of delivering a packet over the backbone network is greater than that over the distribution and access network. Let us assume that the cost of a single link in the backbone, distribution, and access network is $\mathrm{C}_{\mathrm{B}}$, $C_{D}$, and $C_{A}$, respectively. Here, the number of hops inside each area of different network is not taken into account, rather it is embedded in the unit cost of a single link. Note that, the following relation holds: $\mathrm{C}_{\mathrm{B}}>\mathrm{C}_{\mathrm{D}}>\mathrm{C}_{\mathrm{A}}$.

Let us define the following function for the average of the total cost of links in backbone, distribution, and access network by $\mathrm{C}_{\mathrm{BT}}, \mathrm{C}_{\mathrm{DT}}$, and $\mathrm{C}_{\mathrm{AT}}$, respectively, which is given as follows:

$$
\begin{aligned}
& C_{B T}=C_{B} \times L_{C I} \\
& C_{D T}=C_{D} \times L_{I L} \\
& C_{A T}=C_{A} \times L_{L U}
\end{aligned}
$$

\section{Numerical results}

Let us assume that $\mathrm{K}=30, \mathrm{M}=100, \mathrm{k}_{1}=33, \mathrm{k}_{2}=66$, $\mathrm{C}_{\mathrm{B}}=5, \mathrm{C}_{\mathrm{D}}=3$, and $\mathrm{C}_{\mathrm{A}}=1$. Note that the cost is defined in a unitless manner, via which one can define the unit of cost without any constraint.

In order to investigate the validity of the proposed model, let us compare the cost of the proposed power law model with a reference model without power law (video programs are not sorted by 
popularity and they are located arbitrarily) where $33 \%$ and $66 \%$ of video programs are located at LS and IS, respectively, whereas the whole programs are located at CS.

Fig.3 illustrates the cost incurred at the access link. As one can see from Fig. 3 , the cost at the access network is the same for the two models. Fig.4 and Fig.5 illustrates the cost incurred at the distribution and backbone network, respectively.

Summarizing the numerical experiments, we could obtain the following results: First, we could save the link resource of the distribution and backbone network by introducing the power law in the location of the video programs at a hierarchical storage system. Second, resulting from the saving of the link in the distribution and backbone network, we could save the cost of using the link in the end-to-end path of the video stream. This results in a very effective management of the $\mathrm{VoD}$ system with lower cost as compared with the conventional VoD system that does not apply the power law.

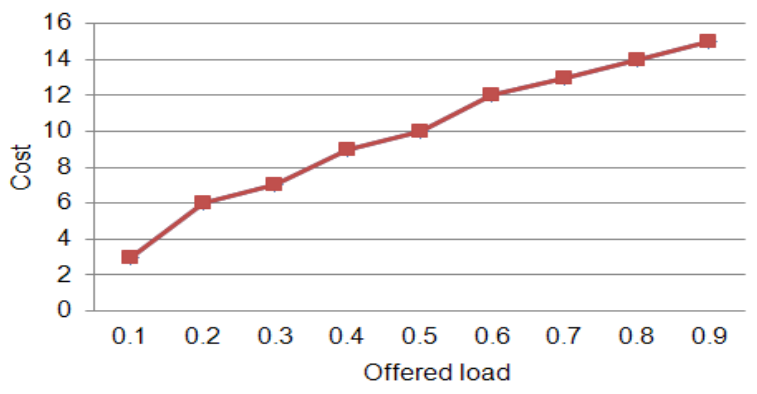

Fig. 3. Cost at access network

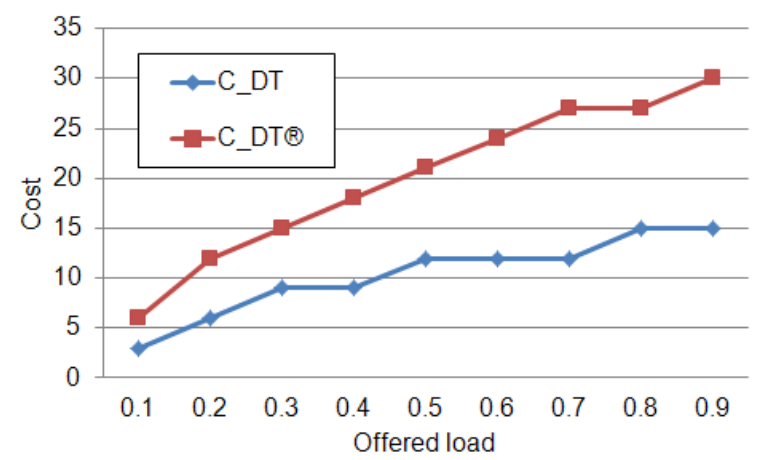

Fig. 4. Cost at distribution network

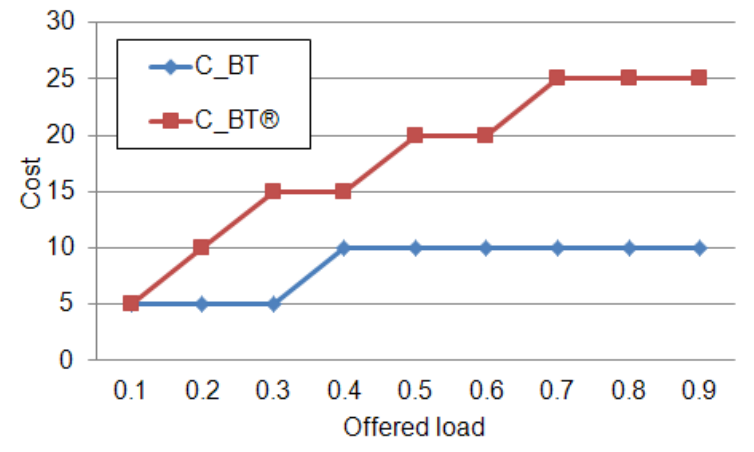

Fig. 5. Cost at core network

\section{Conclusion}

In this work we have proposed an analytic framework to investigate the power of power law in the design of VoD service over an IP network. The novelty of this work can be summarized as follows: First, we exploited the power law into the development of an analytic model to estimate the required capacity of a link for the distributed storage system of VoD. Second, we showed the power of power law in the location of the video contents and its resulting impact on the cost of the service for VoD. Third, we showed a practical result for the link dimensioning and cost performance by using a real-field data for the popularity of the viewing pattern about VoD service.

The implication of this work can be summarized as follows: First, ISPs can apply the proposed method to the design of the network resource for VoD service such as the location and link dimensioning. Second, one can extend the performance evaluation for much greater scale of network and customers by using the proposed framework, which will be practical to the application of this method to the commercial network for $\mathrm{VoD}$ service.

Our future research work includes the accumulation of the real-field data in more extensive scale, via which we can find a much more clear view of the property of power law in the viewing pattern for VoD service. We will also devise a more sophisticated method to determine the number of contents that is located in each area. A new model for the determination of the elements that is related 
to the cost has to be investigated, too.

\section{Acknowledgments}

The author wishes to thank the anonymous reviewers for their constructive comments on this work.

\section{References}

[1] L. A. Adamic and B. A. Huberman, "Zipf's law and the Internet," Glottometrics, vol. 3, no. 2002, pp. 143-150, 2002.

[2] L. Breslau, P. Cao, L. Fan, G. Phillips, and S. Shenker, "Web caching and Zipf-like distributions: evidence and implications," in Proc. IEEE INFOCOM '99, vol. 1, pp. 126-134, New York, U.S.A., Mar. 1999.

[3] M. Mitzenmacher, "A brief history of generative models for power law and lognormal distribution," Internet Math., vol. 1, no. 2, pp. 226-251. Jan. 2011.

[4] A. Mislove, M. Marcon, K. P. Gummadi, P. Druschel, and B. Bhattacharjee, "Measurement and analysis of online social networks," in Proc. ACM SIGCOM IMC '07, pp. 29-42, San Diego, U.S.A., Oct. 2007.

[5] P. Gill, M. Arlitt, Z. Li, and A. Mahanti, "Youtube traffic characterization: a view from the edge," in Proc. ACM SIGCOMM IMC '07, pp. 15-28, San Diego, U.S.A., Oct. 2007.

[6] M. Cha, P. Rodriguez, J. Crowcroft, S. Moon, and X. Amatriain, "Watching television over an IP network," in Proc. ACM SIGCOMM IMC '08, pp. 71-84, Vouliagmeni, Greece, Oct. 2008.

[7] Y. J. Won, M. J. Choi, B. C. Park, W. K. Hong, H. W. Lee, C. G. Hwang, and J. H. Yoo, "Measurement of IPTV traffic in the broadband access network," in Proc. KNOM 2008, TS5-4, Changwon, Korea, Apr. 2008.

[8] H. Lee, "A practical network design for $\mathrm{VoD}$ services," J. KICS, vol. 34, no. 3, pp. 225-234, Mar. 2009.

[9] G. O'driscoll, Next generation IPTV services and technologies, John Wiley \& Sons, 2008.

[10] Y.-C. Lai, Y.-D. Lin, and H.-Z. Lai, "A hierarchical network storage architecture for video-on-demand services," IEEE Trans. Broadcast., vol. 43, no. 2, pp. 145-154, June 1997.

[11] A. Mahanti, N. Carlsson, A. Mahanti, M. Arlitt, and C. Williamson, "A tail of the tails: Power-laws in internet measurements," IEEE Network, vol. 27, no. 1, pp. 59-64, Jan.-Feb. 2013.

[12] H. Lee, Professor Lee's Lectures on Probability, HongPub, 2009.

[13] SK Broadband, "Statistics for the download of the IPTV service," Courtesy of SK Broadband, Aug.-Sep. 2012.

[14] W. Lee, Curve fitting in Microsoft Excel, Retrieved Apr 7, 2013 from http://www.csupomona.edu.

[15] M. S. Allen, B. Y. Zhao, and R. Wolski, "Deploying VoD services on cable networks", in Proc. IEEE ICDCS '07, pp. 63, Toronto, Canada, June 2007.

[16] H. Lee, Fundamentals on queuing system, Bumhanbook, 2011.

[17] H. Lee and K. Sohraby, "Flow-aware link dimensioning for guaranteed-QoS services in broadband convergence networks," $J$. Commun, Networks, vol. 8, no. 4, pp. 410-421, Dec. 2006.

[18] S. Spagna, M. Liebsch, R. Baldessari, S. Niccolini, S. Schmid, R. Garroppo, K. Ozawa, and J. Awano, "Design principles of an operator-owned highly distributed content delivery network," IEEE Commun. Mag., vol. 51, no. 4, pp. 132-140, Apr. 2013. 


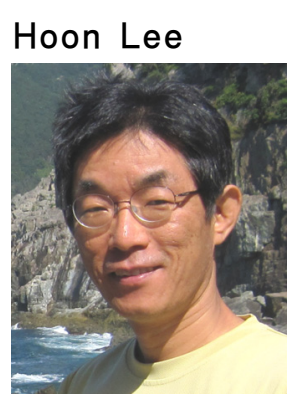

1984 B. E. from Kyungpook

National University (KNU)

1986 M. E. from KNU

1996 Ph.D. from Tohoku

University, Japan.

1986 2001 KT R\&D Center

2001 Changwon National

University

2005 2006 Visiting@U. of Missouri-Kansas City

2011 2012 Visiting@ Marquette University

$<$ Research fields $>$ Design, performance evaluation, traffic engineering, QoS, and charging of computer network 